\title{
Activity-Dependent Feedforward Inhibition Modulates Synaptic Transmission in a Spinal Locomotor Network
}

\author{
David Parker \\ Department of Zoology, University of Cambridge, Cambridge, CB2 3EJ, United Kingdom
}

The analysis of synaptic properties in neural networks has focused on the properties of individual synapses. As a result, little is known of how neural assemblies arise from the connectivity and functional properties of different classes of network neurons.

I examined synaptic properties in the lamprey locomotor network. Here I show that, in addition to their monosynaptic inputs to motor neurons, a proportion of the excitatory network interneurons (EINs) evoke an activity-dependent disynaptic feedforward inhibitory input. Connections from the excitatory interneurons to small ipsilateral inhibitory interneurons were found that could account for the feedforward inhibition. Both synapses in the disynaptic pathway exhibited activity-dependent facilitation during physiologically relevant spike trains, which could contribute to the delayed, activity-dependent development of the feedforward IPSP. Although it was not as common as the feedforward inhibition, the excitatory interneurons could also evoke feedforward excitatory inputs in motor neurons.

EIN inputs to motor neurons usually depress during spike trains. In connections in which a delayed IPSP occurred, blocking the feedforward inhibition in motor neurons or preventing the activation of the disynaptic pathway abolished the depression of the direct EPSP during the spike train and could reveal an underlying facilitation. The feedforward inhibition thus heterosynaptically depressed the direct excitatory input to motor neurons. Activity-dependent heterosynaptic effects acting within network cellular assemblies can thus influence the integration of synaptic inputs in motor neurons. This could help to terminate ipsilateral motor neuron spiking during network activity.

Key words: network; spinal; synaptic; plasticity; lamprey; feedforward

\section{Introduction}

To understand a neural network, information is required on the network architecture and on the cellular and synaptic mechanisms that generate network activity (Selverston, 1980; Getting, 1989; Marder and Calabrese, 1996). A large number of cellular and synaptic mechanisms have been examined in different networks (Marder and Calabrese, 1996). Short-term activitydependent plasticity was originally included among the potential synaptic mechanisms for patterning rhythmic network activity (Selverston, 1980). However, it is only relatively recently that the role of this effect has been addressed (Nadim and Manor, 2000).

I examined activity-dependent synaptic plasticity in the lamprey locomotor network (Parker, 2000). This network consists of hemisegmental networks on the left and right sides of the spinal cord that are coupled by reciprocal inhibitory and excitatory connections. The locomotor network is repeated in each of the $\sim 100$ spinal cord segments. The activity in these segmental networks is coordinated by intersegmental neurons (Hill et al., 2003).

Synaptic transmission can be examined in the lamprey by making paired recordings from identified monosynaptically connected interneurons and motor neurons (Parker, 2000). The

\footnotetext{
Received Sept. 1, 2003; revised 0ct. 1, 2003; accepted 0ct. 1, 2003.

This work was supported by The Royal Society, The Wellcome Trust, and the Biotechnology and Biological Sciences Research Council.

Correspondence should be addressed to David Parker, Department of Zoology, Cambridge University, Downing Street, Cambridge, CB2 3EJ, UK. E-mail: djp27@cam.ac.uk.

Copyright $\odot 2003$ Society for Neuroscience $\quad$ 0270-6474/03/2311085-09\$15.00/0
}

analysis of identified connections has shown that synapsespecific activity-dependent plasticity can develop during physiologically relevant spike trains (Parker, 2000, 2003). It could thus occur during network activity to influence the patterning of the network output. However, the data have primarily been obtained at interneuron connections to motor neurons (Parker, 2000, 2003), and, because motor neurons are not considered to be part of the locomotor network (Wallén and Lansner, 1984) (but see Buchanan et al., 1998), the potential role of activity-dependent plasticity in patterning network activity has not been addressed. Connections between segmental network interneurons thus must be examined.

The analysis of connections between network interneurons is complicated by the requirement of making paired recordings from relatively small cells and also by uncertainty over the identify of interneurons in the segmental network. The uncertainty particularly relates to the interneurons that connect hemisegmental networks on the left and right sides of the spinal cord (Parker, 2000). As a result, I focused on connections between the excitatory interneurons (EINs) and small ipsilateral inhibitory interneurons (SiINs) in the hemisegmental network, because information is available that suggests that these cells are components of the segmental network (Buchanan and Grillner, 1988; Buchanan et al., 1989).

The analysis in this study was prompted by the observation that intracellular stimulation of an EIN can evoke activitydependent feedforward inhibition in motor neurons (Parker, 
2003). Feedforward inhibition is a common phenomenon that has been identified in spinal, cortical, and hippocampal networks (Eccles et al., 1954; Buszaki, 1984; Miles, 1990). EIN inputs to the SiINs were identified that provided the appropriate disynaptic circuit needed to evoke the feedforward inhibition. The activitydependent properties of synapses in this pathway were consistent with the activity-dependent development of the feedforward inhibition. The feedforward inhibition heterosynaptically depressed the direct excitatory input to motor neurons. Activitydependent plasticity acting within specific neural assemblies could thus influence the integration of synaptic inputs in motor neurons.

\section{Materials and Methods}

Adult male and female lampreys (Lamptera fluviatilis) were anesthetized with MS-222, and the spinal cord and notochord were removed. The spinal cord was isolated from the notochord and placed ventral side up in a Sylgard-lined chamber and superfused with Ringer's solution containing the following (in mM): $138 \mathrm{NaCl}, 2.1 \mathrm{KCl}, 1.8 \mathrm{CaCl}_{2}, 1.2 \mathrm{MgCl}_{2}, 4$ glucose, 2 HEPES, and 0.5 L-glutamine. The Ringer's solution was bubbled with $\mathrm{O}_{2}$, and the $\mathrm{pH}$ was adjusted to 7.4 with $1 \mathrm{M} \mathrm{NaOH}$. The experimental chamber was kept at a temperature of $10-12^{\circ} \mathrm{C}$. Calcium was reduced to $50 \%$ in low-calcium Ringer's solution and increased to $200 \%$ in high-calcium Ringer's solution. The analysis shown here is based on recordings from 192 animals.

Synaptic plasticity was examined by making paired intracellular recordings from physiologically identified network neurons (Buchanan, 1993) using thin-walled micropipettes filled with 3 м potassium acetate and $0.1 \mathrm{~m}$ potassium chloride. Motor neurons were identified by recording orthodromic extracellular spikes in the associated ventral root after current injection into their somata. EINs and SiINs were identified by their ability to elicit monosynaptic EPSPs or IPSPs, respectively (Buchanan and Grillner, 1988; Buchanan et al., 1989). The SiINs could be distinguished from the lateral inhibitory interneurons (LINs), because the LINs have large cell bodies on the lateral margin of the gray matter (Rovainen, 1974). Connections between interneurons were examined by first making a paired recording from a presynaptic EIN or SiIN (interneuron $_{1}$ ) and a postsynaptic motor neuron or unidentified neuron. The postsynaptic electrode was then withdrawn and used to find interneurons that synapsed onto interneuron ${ }_{1}$. All presynaptic interneurons were located one to two segments rostral to postsynaptic interneurons, and in the same segment or one segment rostral to postsynaptic motor neurons. Monosynaptic connections were identified by their reliability and constant latency after presynaptic stimulation at $20 \mathrm{~Hz}$. The membrane potential in control and in drugs or altered Ringer's solutions was kept constant by injecting depolarizing or hyperpolarizing current using single-electrode discontinuous current clamp. The membrane potential of the EINs and SiINs tended to be more depolarized ( -50 to $-55 \mathrm{mV}$ ) than the membrane potential of motor neurons (usually less than -60 $\mathrm{mV}$ ), presumably attributable to damage to the smaller interneurons. All cells were tonically hyperpolarized to between -65 to $-70 \mathrm{mV}$ during experiments. Glass suction electrodes were placed bilaterally on the spinal cord caudal to the cells recorded from to record extracellular activity in ipsilateral and contralateral axons (see Fig. 2 Aii). The suction electrode tips covered the entire left or right half of the spinal cord. An Axoclamp 2A amplifier (Axon Instruments, Foster City, CA) was used for voltage recording and current injection. Data were acquired, stored, and analyzed on a computer using an analog-to-digital interface (Digidata 1200; Axon Instruments) and Axon Instruments software (pClamp 8).

Activity-dependent synaptic plasticity was examined by stimulating the presynaptic interneuron at frequencies of 5, 10, and $20 \mathrm{~Hz}$. These frequencies are within the range reported of interneuron spiking during network activity (Buchanan and Cohen, 1982; Buchanan and Kasicki, 1995). Three to 20 spike trains were evoked at $30 \mathrm{sec}$ intervals at each frequency. In some cases, the initial postsynaptic potentials in the spike trains were used as a measure of low-frequency-evoked inputs. Twenty spikes were evoked in each train. Although this exceeds the upper number of spikes that interneurons generate during network activity, network relevant plasticity occurred over the first five spikes (Buchanan and Cohen, 1982; Buchanan and Kasicki, 1995). Longer spike trains are used because the inputs over the latter part of the spike train can subsequently provide insight into the mechanisms underlying the plasticity (Parker, 2003).

EPSP or IPSP amplitudes were measured as the peak amplitude above or below the baseline immediately preceding the postsynaptic potential. At the frequencies used, there was usually little summation of the postsynaptic potentials. Statistical significance was examined using twotailed paired or independent $t$ tests or one-way ANOVA. $n$ refers to the number of connections examined. Several connections could be examined in a single animal. All values given refer to mean \pm SEM.

\section{Results}

\section{EIN inputs evoke biphasic responses in motor neurons}

The EINs provide glutamatergic excitation in the hemisegmental network (Buchanan, 2001). The analysis of a large sample of EIN inputs to motor neurons $(n=278)$ has shown that this connection has variable activity-dependent properties (Parker, 2003): $\sim 50 \%$ of connections depressed and $\sim 30 \%$ facilitated. The remaining connections were either biphasic (facilitation followed by depression) or showed no plasticity over the spike train. This variability occurred when inputs from several EINs were examined in a single motor neuron (Parker, 2003), suggesting that it was an intrinsic network property and not simply attributable to differences between experiments.

In a proportion of EIN to motor neuron connections $(\sim 10 \%$; $n=27)$, intracellular stimulation of an EIN reliably evoked a biphasic response in motor neurons. This consisted of an initial EPSP followed by a delayed IPSP (Fig. $1 A$ ). In the majority of cases $(n=21)$, a noticeable IPSP had developed between the second and fourth spikes in the train (Fig. 1 $A, B$ ). In these cases, the delayed inhibitory input was frequency dependent; it was either absent, or its amplitude was reduced at lower stimulation frequencies (Fig. 1C,D). In addition to the delayed input during spike trains, in a smaller population of connections $(n=6)$, an IPSP could reliably follow single low frequency $(0.1 \mathrm{~Hz})$ EPSPs (Fig. 2Ai), and a single low-frequency-evoked EPSP could even be followed by several IPSPs $(n=2)$ (Fig. 2 Aii). Intracellular EIN stimulation could also evoke a reliable polysynaptic inhibitory input in the absence of a direct EPSP $(n=3)$ (Fig. $2 \mathrm{Bi})$. In addition to these feedforward inhibitory effects, stimulation of an EIN could also evoke a biphasic $(n=7)$ (Fig. $2 \mathrm{Bii})$ or triphasic $(n=2)$ (Fig. 2 Biii) excitatory input in motor neurons or unidentified neurons. These feedforward effects were presumably evoked by divergent connections from the stimulated EIN that reliably activated interposed inhibitory and excitatory interneurons. Because the biphasic excitatory-inhibitory input (Fig. 1) was the most common effect, it was examined in detail here.

\section{Source of the delayed IPSP}

To confirm that the delayed IPSP was mediated by an interposed inhibitory interneuron, high-calcium Ringer's solution was used to block transmission in polysynaptic pathways (Berry and Pentreath, 1976). Although high-calcium Ringer's solution can increase the amplitude of monosynaptic EIN-evoked EPSPs (Parker, 2003), presumably by increasing the EIN release probability, it blocks polysynaptic inputs by increasing the spike threshold, thus making it more difficult for synaptic inputs to evoke spiking in interposed neurons (Berry and Pentreath, 1976). High-calcium Ringer's solution increased ( $n=4$ of 6 ) or had no effect $(n=2$ of 6$)$ on the amplitude of the initial EIN-evoked EPSP. However, it consistently blocked the delayed IPSP $(n=6$ of 6) (Fig. 3). This result confirmed that the delayed IPSP resulted 

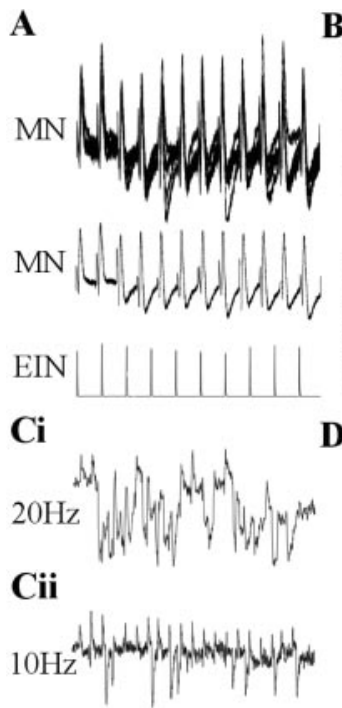

\section{Ciii}

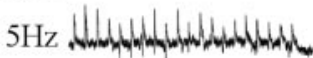

D
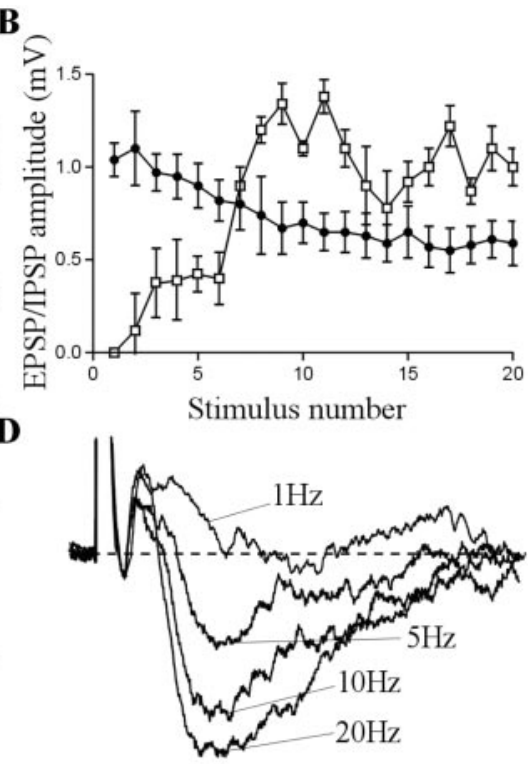

$\begin{array}{rr}\mathrm{A}, \mathrm{D} & 1 \mathrm{mV} \\ \mathrm{C} & 5 \mathrm{mV} \\ 100 \mathrm{mV}\end{array}$

Figure 1. Intracellular stimulation of a single EIN can evoke a biphasic input in motor neurons that consisted of a direct EPSP followed by a delayed IPSP. A, Traces showing the development of a consistent biphasic IPSP over a $20 \mathrm{~Hz}$ spike train. The middle trace is an average of the activity shown above ( $n=20$ sweeps). $B$, Graph showing the amplitude of the monosynaptic EPSP (black circles) and the delayed IPSP (white squares) in motor neurons after stimulation of the presynaptic EIN at $20 \mathrm{~Hz}$ ( $n=21$ connections). (i-Ciii, Traces showing the frequencydependent development of the delayed IPSP in a single connection. In this case, the summation of IPSPs during $20 \mathrm{~Hz}$ stimulation effectively abolished the direct EPSP. The effect was less pronounced at $10 \mathrm{~Hz}$ and was absent at $5 \mathrm{~Hz}$. D, The averaged delayed IPSP over a train of 20 spikes at frequencies of $1,5,10$, and $20 \mathrm{~Hz}$. The lowest voltage calibration in this and subsequent figures refers to the amplitude of action potentials on that figure.

from the EIN-mediated activation of an interposed inhibitory neuron.

There are two potential sources of the delayed IPSP in the hemisegmental network: the glycinergic LINs and the glycinergic SiINs (Buchanan, 2001). The LINs receive monosynaptic inputs from the EINs, and they monosynaptically inhibit the large ipsilateral crossed caudal (CC) interneurons and ipsilateral motor neurons (Rovainen, 1982; Buchanan, 2001). They could thus potentially evoke the delayed feedforward IPSP. LIN axons project ipsilaterally and caudally along the entire length of the spinal cord (Rovainen, 1974). Axonal activity can be recorded extracellularly from the surface of the spinal cord. If an LIN was activated by EIN stimulation, extracellular activity should be recorded ipsilaterally and caudally at some distance from the stimulated EIN. However, the delayed IPSP was not associated with extracellular activity when the recording electrode was placed $\geq 10$ segments ipsilateral and caudal to the stimulated EIN ( $n=8$ of 8 ) (Fig. 2 Aii), thus suggesting against LIN activation. In addition, the LINs are only found in the rostral trunk region of the spinal cord (Rovainen, 1974), but delayed IPSPs could be evoked in caudal trunk regions $(n=7)$. Both of these features suggest against the involvement of the LINs in evoking the delayed IPSP.

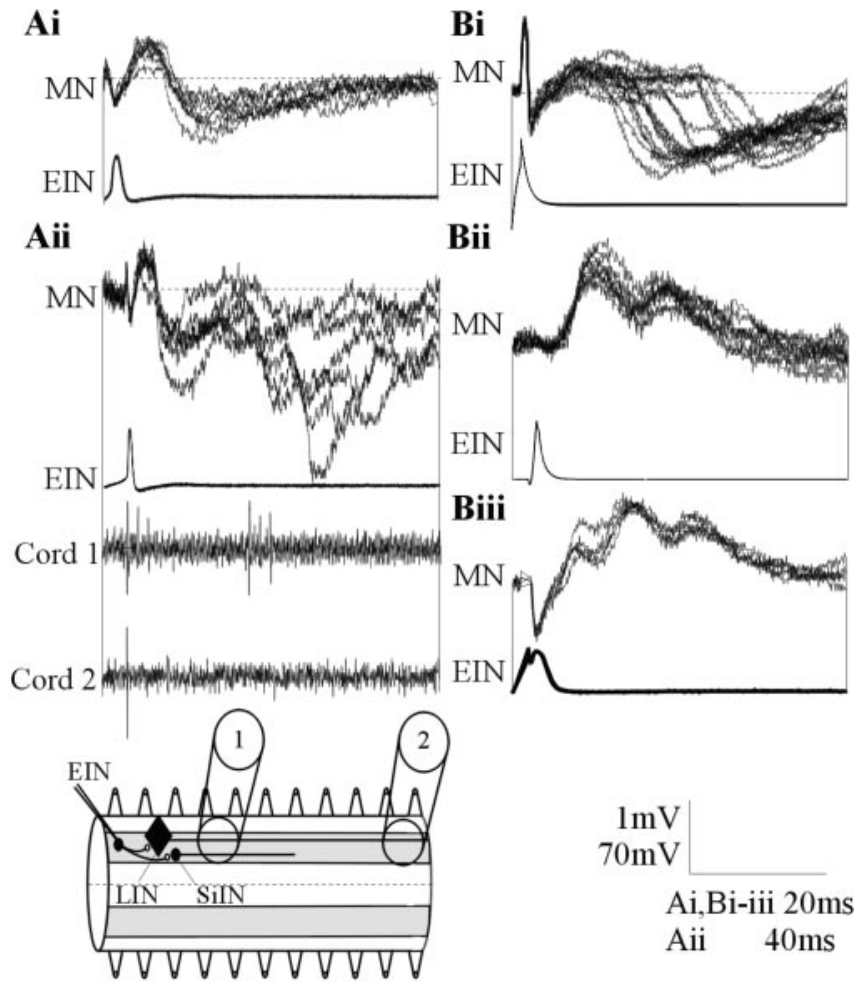

Figure 2. Ai, In addition to the activity-dependent feedforward IPSP during spike trains, in a smaller proportion of connections, a delayed IPSP could consistently follow low-frequency $(0.1$ $\mathrm{Hz}$ )-evoked EPSPs. Aii, A single low-frequency-evoked EPSP could also be followed by several polysynaptic IPSPs. The delayed inhibitory input in this cell was associated with extracellular activity recorded in a suction electrode placed three to four segments caudal to the stimulated EIN but not in a suction electrode placed 10 segments caudal to the EIN. The diagram shows the extracellular recording arrangement and the axonal projections of the LINs and SiINs. The absence of activity in an electrode placed 10 segments caudal to the stimulated EIN suggests against LIN activation. Bi, A delayed IPSP could also develop in the absence of a direct EPSP. EIN stimulation could also result in delayed biphasic (Bii) or triphasic (Biii) excitatory inputs. MN, Motor neuron.

The SiINs are found in rostral and caudal regions of the spinal cord (Buchanan and Grillner, 1988; my unpublished observations), and they make functionally strong monosynaptic connections onto motor neurons (Buchanan and Grillner, 1988). As with the other small segmental network interneurons (Buchanan, 2001), SiIN axons project at most seven segments caudally (my unpublished observations). The role of the SiINs in the feedforward inhibition was supported by recording extracellular activity in suction electrodes placed three to four segments caudal to EINs that evoked delayed inhibitory inputs in four of five experiments (Fig. 2Aii). The conduction velocity of the SiINs was $0.6 \pm 0.3$ $\mathrm{m} / \mathrm{sec}$. This is a similar conduction velocity to the EINs (Buchanan et al., 1989). Although this suggested that the SiINs could have evoked the delayed IPSP, an EIN input to the SiINs that would be needed to evoke the IPSP had not been identified previously. In this study, monosynaptic connections from EINs onto SiINs were found ( $n=7$ of 92 of SiINs in which EIN input were sought) (Fig. 4Ai). Monosynaptic connections from the SiINs to EINs were also found ( $n=3$ of 172 EINs in which SiIN inputs were sought) (Fig. 4Aii), but there were no reciprocal connections between the EINs and SiINs in the small sample of connections examined here $(n=10)$ (Fig. $4 B)$. The hemisegmental network contains more EINs than SiINs (Buchanan, 2001), which, given the limited opportunity to search for presynaptic inputs before the postsynaptic interneuron recording was lost, 


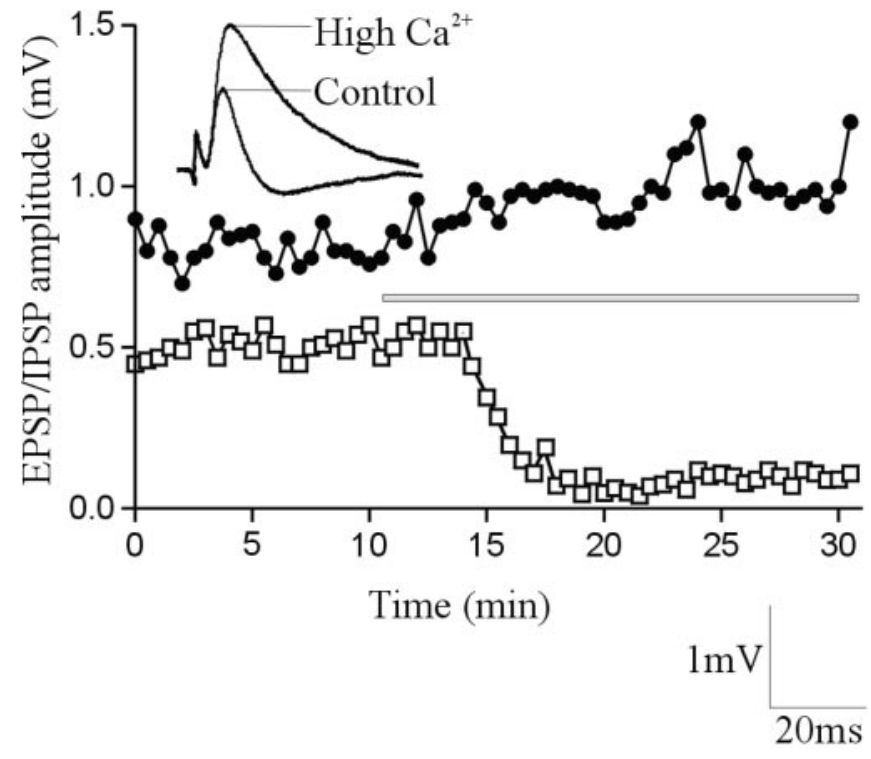

Figure 3. The delayed IPSP was blocked in high-calcium Ringer's solution. The graph shows the amplitude of the direct EPSP (black circles) and the delayed IPSP (white squares) in a single experiment. The bar shows the onset and duration of high-calcium Ringer's solution application. The traces above the graph show an average of the fifth EPSP in a $20 \mathrm{~Hz}$ spike train in control and in the presence of high-calcium Ringer's solution.

presumably accounted for the greater probability of finding an EIN input to an SiIN than an SiIN input to an EIN. The limited opportunity to search for identified presynaptic inputs also means that the small proportion of EIN to SiIN and SiIN to EIN connections ( 8 and $2 \%$, respectively) will almost certainly not reflect the actual proportions of these connections in the locomotor network.

The latency of the synaptic input to SiINs located one to two segments caudal to the stimulated EIN was $5.4 \pm 1.4 \mathrm{msec}$, and the latency of the SiIN input to motor neurons located one segment caudal was $4.2 \pm 0.3 \mathrm{msec}$. These latencies are consistent with monosynaptic connections in the lamprey at the experimental temperatures used (Rovainen, 1974) and when the conduction velocity in the SiINs $(0.6 \pm 0.3 \mathrm{~m} / \mathrm{sec})$ and EINs $(0.7 \pm 0.3$ $\mathrm{m} / \mathrm{sec}$ ) (Buchanan et al., 1989) are taken into account. The inputs also followed reliably when the presynaptic cell was stimulated at $20 \mathrm{~Hz}$, which is indicative of a monosynaptic connection (Berry and Pentreath, 1976). The latency of a delayed IPSP evoked by feedforward activation of the SiINs should thus be between 9 and $10 \mathrm{msec}$. The actual latency was $\sim 12.2 \pm 0.7 \mathrm{msec}$. The difference in the predicted and observed latencies $(\sim 2 \mathrm{msec})$ would probably not allow the presence of another synapse in the feedforward pathway (Rovainen, 1974) but could instead reflect an overestimation of the actual latency attributable to the difficulty in measuring the onset of the IPSP when it was obscured by the direct EPSP.

\section{Synaptic properties in the disynaptic pathway}

The EIN input to the SiINs provided the appropriate network circuitry needed to evoke the delayed IPSP. Although this was obviously an essential requirement, the EIN input still has to be able to activate the interposed neuron if it is to evoke the delayed IPSP. EIN-evoked EPSPs rarely evoke action potentials in motor neurons (Parker, 2003). This suggests that there must be specialized cellular and synaptic properties in the disynaptic pathway that enabled the EIN input to activate the interposed inhibitory interneuron. The amplitude of EIN-evoked EPSPs in the SiINs was $3.4 \pm 0.3 \mathrm{mV}(n=7)$. This is significantly larger than the amplitude of EIN-evoked EPSPs in motor neurons or other EINs $(p<0.05)$ (Fig. 4C). The half-width of EIN-evoked EPSPs in the SiINs was also significantly longer than in motor neurons (12.1 \pm 0.5 and $10.2 \pm 0.4 \mathrm{msec}$, respectively; $p<0.05$ ) (Fig. $5 A$ ). The EIN-evoked EPSP in the SiINs usually facilitated ( $n=6$ of 7 ) (Fig. $5 A, B)$. This facilitation could contribute to the delayed activation of the interposed inhibitory interneuron and thus to the delayed development of the feedforward IPSP. Significant facilitation of the EIN-evoked EPSP occurred at $20 \mathrm{~Hz}(180 \pm 17 \%$ of control; $p<0.01)$ but not at 10 or $5 \mathrm{~Hz}(120 \pm 17$ and $105 \pm 19 \%$, respectively; $p>0.05$; data not shown). This is consistent with the frequency dependence of the delayed IPSP (Fig. 1C,D).

The facilitation of a large initial EPSP will make the EIN input to the SiINs functionally stronger than the EIN input to motor neurons, in which depression usually develops from a smaller initial EPSP (Fig. 5A,B) (Parker, 2003). The functional strength of the connection was supported by the ability of EIN-evoked EPSPs to evoke spikes in a relatively high proportion of SiINs (57\%; $n=4$ of 7) (Fig. 5C) compared with motor neurons (3\%; $n=8$ of 277) (Parker, 2003). Reliable spiking in the SiINs usually occurred on the second to third EPSPs in the train $(n=3)$ (Fig. $5 C)$. This also contrasts with the EIN input to motor neurons, in which, if spiking occurred, it tended to be evoked reliably by all of the EPSPs in the spike train (Parker, 2003). All of the SiINs were routinely current clamped to between -65 and $-70 \mathrm{mV}$ (see Materials and Methods), and the actual resting membrane potential of the cells in which spikes were or were not evoked did not differ ( $55 \pm 4 \mathrm{mV}, n=4$ and $-54 \pm 6 \mathrm{mV}, n=3$, respectively). This suggests that the spiking did not simply reflect a relatively depolarized membrane potential in some SiINs. The amplitude of the initial EIN-evoked EPSP also did not differ in SiINs that spiked or did not spike ( $3.5 \pm 0.7$ and $3.4 \pm 0.9 \mathrm{mV}$, respectively).

A functionally strong connection is also suggested at the second synapse in the proposed disynaptic pathway, the SiIN input to motor neurons. SiIN inputs to motor neurons usually depressed during spike trains (47\% depressed, 32\% facilitated, 2\% biphasic, and $18 \%$ unchanged) (Fig. $5 G$ ). However, six of the seven SiINs that received identified inputs from EINs made facilitating connections onto motor neurons (Fig. 5D-F). Interestingly, the SiINs that facilitated were the cells that received facilitating EIN inputs; the SiIN input to the postsynaptic motor neuron depressed when it received a depressing EIN input (Fig. $5 F)$. The facilitation of the SiIN input was greater at $20 \mathrm{~Hz}(187 \pm$ $11 \%$; $p<0.05)$ than at $10 \mathrm{~Hz}(153 \pm 12 \%$; $p<0.05)$ or $5 \mathrm{~Hz}$ $(111 \pm 7 \% ; p>0.05)$. This effect could also contribute to the frequency dependence of the delayed IPSP (Fig. 1C,D).

\section{The disynaptic pathway heterosynaptically depressed the direct input}

The presence of a delayed IPSP could influence the integration of synaptic inputs in motor neurons. The functional effect of the delayed IPSP was initially examined by blocking the output of the disynaptic inhibitory pathway with strychnine. This blocks inhibitory inputs in lamprey (McPherson et al., 1994) (Fig. 6A,B). Although it is associated with effects on glycinergic transmission, strychnine can also affect GABAergic inhibition in lamprey and mammalian systems (Safronov et al., 1989; Jonas et al., 1998). In the connections that were examined, the monosynaptic EINevoked EPSP was either depressed $(n=4)$ or unchanged $(n=2)$ over the spike train, and a noticeable delayed IPSP had developed between the second and fourth EPSPs. Strychnine ( $1 \mu \mathrm{M})$ consis- 


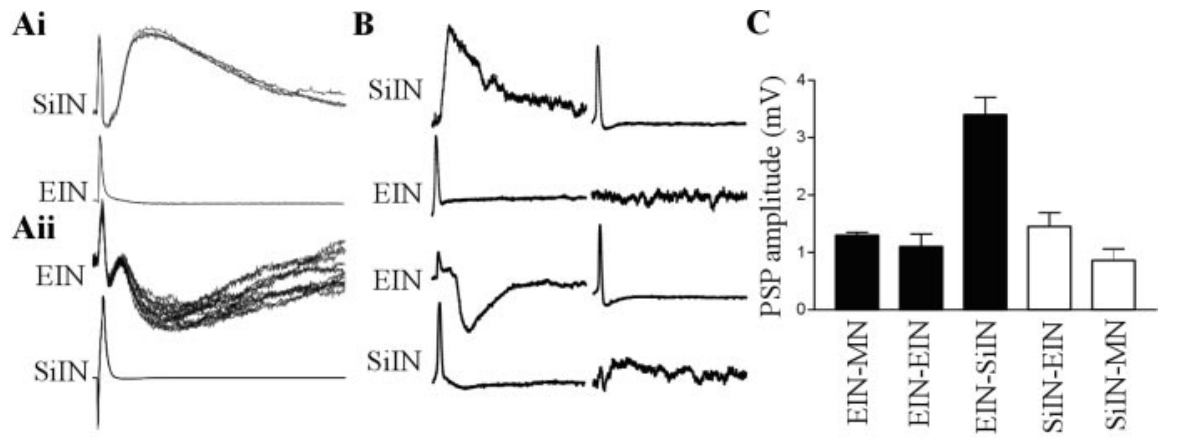

Figure 4. Ai, EINs make monosynaptic connections onto SilNs. Traces showing five overlaid monosynaptic EPSPs evoked at 0.1 $\mathrm{Hz}$. Aii, SilNs can also make monosynaptic inhibitory connections onto the EINs. Twenty overlaid IPSPs evoked at $0.1 \mathrm{~Hz}$ are shown. $B$, Traces showing the lack of reciprocal connections between EINs and SilNs in this study. Each trace is an average of 5-10 sweeps at $0.1 \mathrm{~Hz}$. The small depolarizing evoked in the SilN by the EIN that received the monosynaptic SilN input did not persist when the connection was stimulated at $20 \mathrm{~Hz}$, and thus it was presumably not monosynaptic. C, Bar graph comparing the amplitude of low-frequency-evoked monosynaptic postsynaptic potentials. Note that the EIN-evoked EPSP in the SilNs is significantly larger than other postsynaptic potential amplitudes (EIN-MN, $n=278 ;$ SilN-MN, $n=92 ;$ EIN-SilN, $n=7$; SilN-EIN, $n=3$; EIN-EIN, $n=11)$. MN, Motor neuron.

tently blocked the delayed IPSP ( $n=6$ of 6 ). This resulted in an increase in the amplitude and half-width of the monosynaptic EPSP (Fig. $6 B, E, F$ ). The effects of strychnine were greatest during the spike train (Fig. 6B), which supported a delayed influence of the IPSP. In addition, blocking the delayed IPSP converted the synapse from depressing or unchanged into facilitating $(n=6$ of 6) (Fig, 6B,E,F), suggesting that it had an activity-dependent influence on the direct EPSP. The EINs typically depress during spike trains (Parker, 2003). Although the above analysis shows that depression an be caused by the feedforward IPSP, this effect cannot account for the depression in all connections, because depression is seen in the presence of strychnine (Parker and Grillner, 2000).

In addition to blocking its output, the influence of the disynaptic pathway was also examined by preventing the activation of the interposed inhibitory interneuron and thus functionally disconnecting the disynaptic pathway (Fig. 6A). As shown above, the delayed IPSP was blocked in high-calcium Ringer's solution, presumably because the spike threshold was increased (Berry and Pentreath, 1976), and thus it was harder for EIN-evoked EPSPs to evoke spiking in interposed interneurons. High-calcium Ringer's solution usually increased the amplitude of the initial EPSP $(n=$ 4 of 6), probably attributable to an increased release probability, and increased the EPSP half-width $(n=5$ of 6 ) (Fig. $6 C, E)$. These effects were also greater during the spike train, again supporting a delayed influence of the IPSP. High-calcium Ringer's solution also abolished the depression of the EPSP during the spike train. Because an increase in release probability should increase the depression of the EIN input to motor neurons (Parker, 2003), the reduced depression will again reflect the removal of the delayed IPSP. The increased release probability could, however, have reduced any facilitation of the direct EIN input to motor neurons.

The effect of disconnecting the disynaptic pathway was examined further using low-calcium Ringer's solution $(n=3$; data not shown) or the non-NMDA receptor antagonist DNQX (2 $\mu \mathrm{M}$; $n=4$ ) (Alford and Grillner, 1990) to reduce the amplitude of glutamatergic EPSPs $(n=4)$ (Fig. $6 D-F)$ or the NMDA receptor $\begin{aligned} & 2 \mathrm{mV} \\ & 60 \mathrm{mV} \\ & \text { Ai,Aii } 15 \mathrm{~ms} \\ & \mathrm{~B} \quad 25 \mathrm{~ms}\end{aligned}$

antagonist AP-5 $(100 \mu \mathrm{M})$ to abolish the NMDA component of the EPSP $(n=3)$ (Alford and Grillner, 1990) (data not shown). All of these treatments reduced the amplitude of the direct EIN-evoked EPSP in motor neurons by $30-70 \%$ (Fig. $6 D)$. Although not studied in detail, preliminary results suggest that the amplitude of EIN-evoked EPSP in the SiINs was reduced to a similar extent by low-calcium Ringer's solution ( $43 \%$ of control; $n=2$ ) or $2 \mu \mathrm{M}$ DNQX (52\% of control; $n=2$; data not shown). These treatments all blocked the delayed IPSP, presumably because the reduced amplitude of the EINevoked EPSP meant that it was unable to activate the interposed inhibitory interneuron. The removal of the delayed IPSP again increased the half-width of the direct EPSP and converted the depression of the monosynaptic EPSP into facilitation (Fig. $6 D-F)$. Because the facilitation of EINevoked EPSPs usually develops from lower initial release probabilities (Parker, 2003), the facilitation in low-calcium Ringer's solution could simply have reflected a reduction in release probability and not the removal of the feedforward inhibitory input. However, glutamate receptor antagonists will reduce the amplitude of the postsynaptic response to glutamate and should not affect the presynaptic release probability. Glutamate receptors can be located presynaptically on descending reticulospinal axons in the lamprey, in which they facilitate glutamate release (Cochilla and Alford, 1999). If anything, glutamate receptor antagonists should thus have enhanced the depression of the direct EPSP, not reduced it. The conversion of depression into facilitation in these experiments again presumably reflects the removal of the feedforward inhibition

\section{Properties of the feedforward excitatory input}

As shown above, stimulation of a single EIN could also evoke a biphasic $(n=7)$ (Fig. 2 Bii) or occasionally a triphasic $(n=2)$ (Figs. 2 Biii, $7 B$ ) excitatory input in motor neurons. These inputs presumably reflect the presence of functionally strong divergent connections from the stimulated EIN onto other EINs (Fig. 7A) (Parker and Grillner, 2000). Although this effect occurred less frequently than the feedforward inhibition ( $n=9$ of 278), its properties were also examined.

Delayed EPSPs occurred after low-frequency $(0.1 \mathrm{~Hz})$-evoked EPSPs in a higher proportion of connections ( $n=7$ of $9 ; 78 \%)$ than the delayed IPSP ( $n=6$ of $27 ; 22 \%$ ). The amplitude of the biphasic EPSP could facilitate during spike trains at frequencies of $5-20 \mathrm{~Hz}$ (Fig. 7D), but the triphasic input was variable and usually failed, particularly at higher stimulation frequencies (Fig. $7 B, C)$.

The biphasic EPSP had a latency of $11.8 \pm 1.8 \mathrm{msec}$. This is again consistent with a disynaptic pathway (see above). However, in contrast to the delayed IPSP, the delayed EPSP was primarily resistant to glutamate receptor antagonists $(n=4)$ or high- or low-calcium Ringer's solution $(n=5)$. High-calcium Ringer's solution usually increased the monosynaptic EPSP amplitude $(n=3$ of 4$)$. Although the reliability of the delayed EPSP was reduced in high-calcium Ringer's solution ( $n=4$ of 4 ), it was not necessarily blocked (Fig. 7Di). The delayed EPSP also persisted 
A
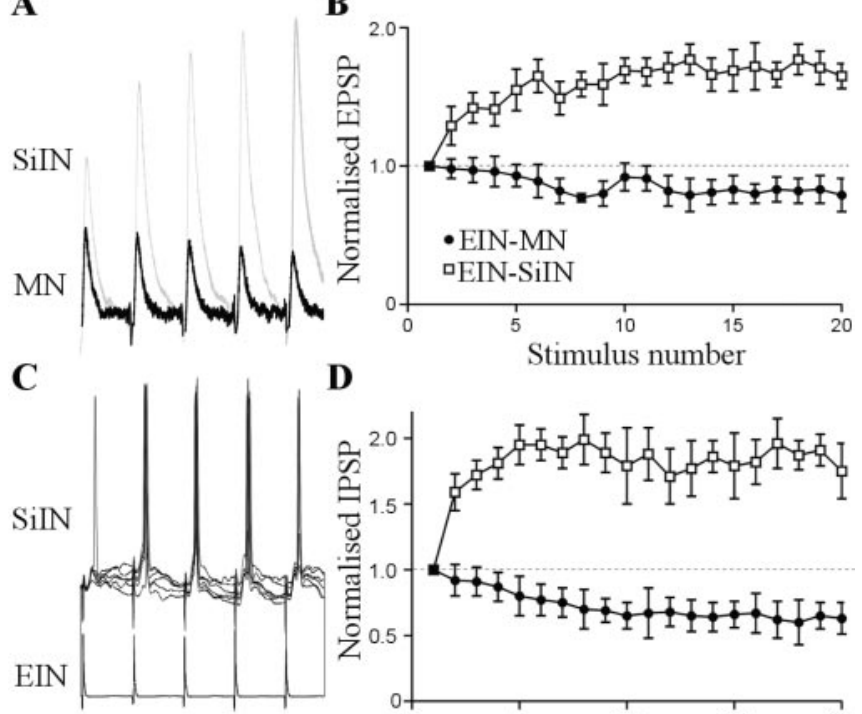

D

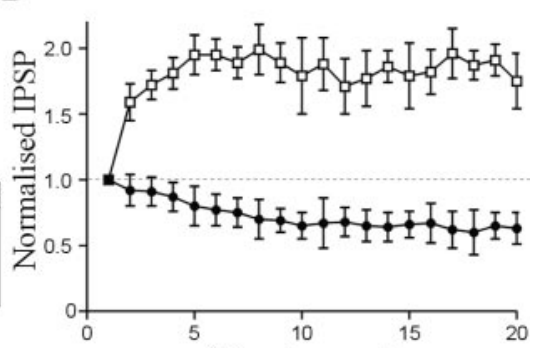

E $\quad$ F

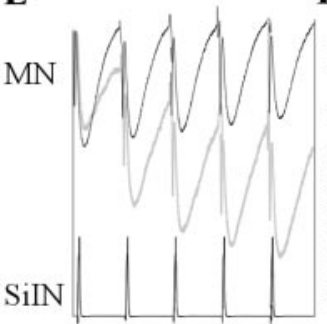

F

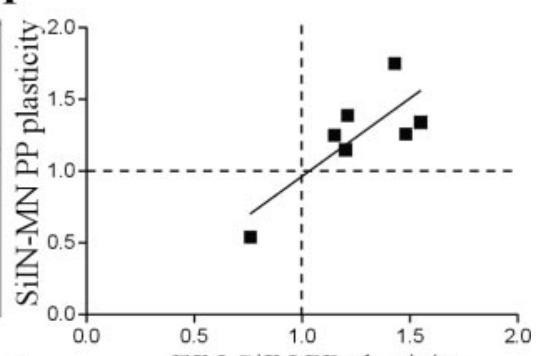

G
A

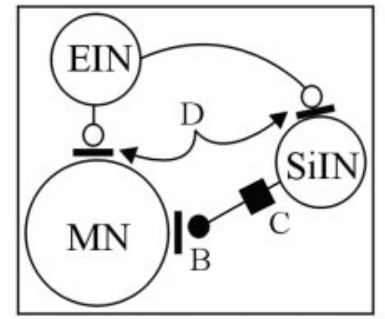

C

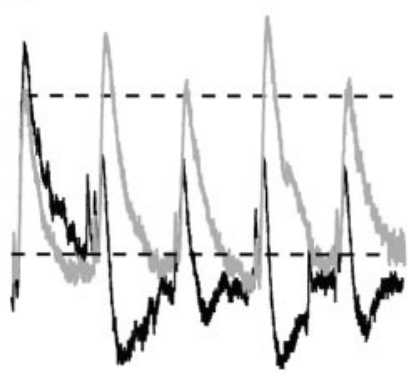

B

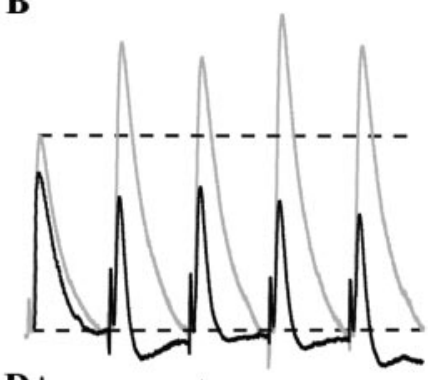

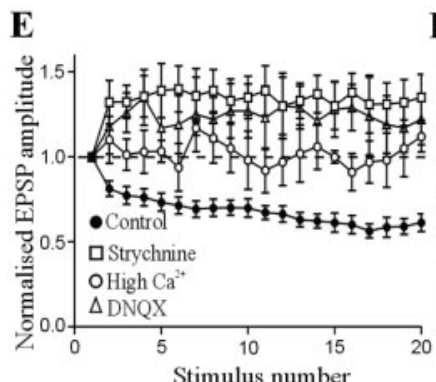

Stimulus number

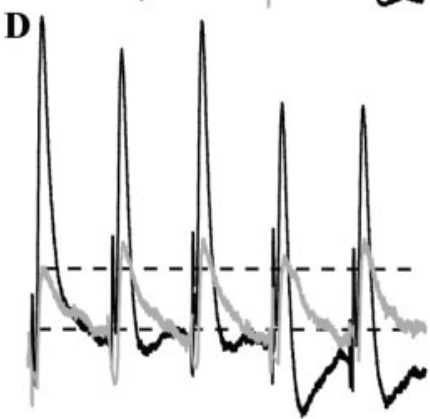

$\mathbf{F}$

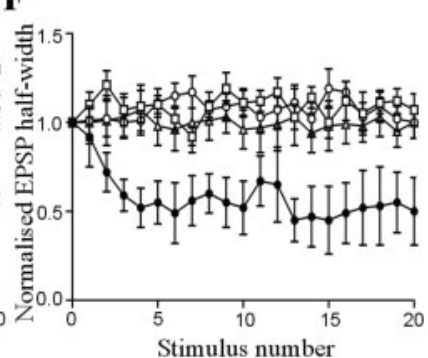

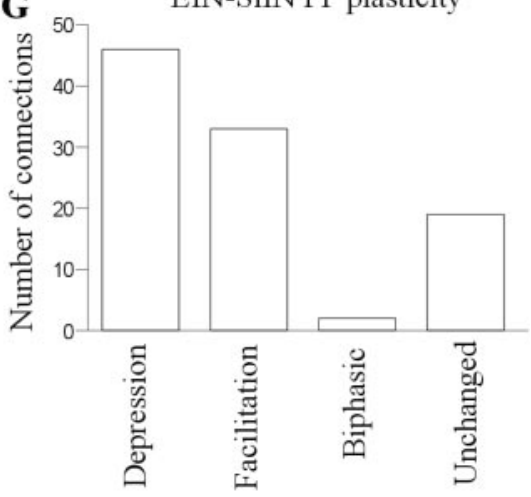

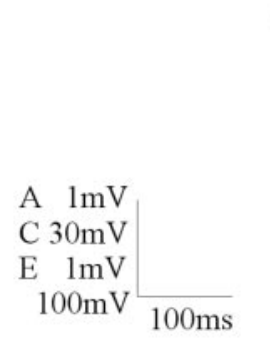

Figure 5. Synaptic properties in the putative disynaptic pathway. A, EIN inputs usually facilitate during $20 \mathrm{~Hz}$ spike trains in the SilNs but usually depress in motor neurons (MN). The input from two separate EINs is shown. $B$, Graph showing the activity-dependent plasticity of EIN inputs to SilNs ( $n=7$; white squares) and EIN inputs to motor neurons ( $n=225$; black circles) after presynaptic stimulation at $20 \mathrm{~Hz}$. C, EIN-evoked EPSPs can evoke spiking in the SilNs. The spiking occurred reliably during the spike train but not usually on the initial EPSP. D, Graph showing the activity-dependent plasticity of SilN inputs to motor neurons that received identified EIN inputs ( $n=7$; white squares) and of SilNs that did not have an identified EIN input ( $n=$ 85; black circles). E, SilN inputs to motor neurons usually depressed, but SilNs that received identified facilitating inputs from EINs made facilitating connections onto motor neurons (gray trace). $F$, Graph showing the correlation of paired pulse (PP) plasticity $\left(\mathrm{PSP}_{2} / \mathrm{PSP}_{1}\right)$ of EIN inputs to SilNs and SilN inputs to motor neurons. G, Graph showing the distribution of different types of activity-dependent plasticity of SilN inputs to motor neurons in response to $20 \mathrm{~Hz}$ spike trains $(n=92)$.

when the release probability was reduced in low-calcium Ringer's solution (Fig. 7Di), and, in zero-calcium Ringer's solution, the delayed EPSP persisted longer than the direct EPSP ( $n=3$ of 4$)$ (Fig. 7Dii). The reduced sensitivity of the delayed EPSP to zero-

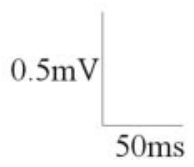

Figure 6. The functional effects of the delayed IPSP in motor neurons. A, Summary diagram showing the proposed circuitry underlying the direct EPSP and delayed IPSP and the methods used to examine the influence of the delayed IPSP. Open circles are excitatory inputs, and filled circles are inhibitory inputs. The letters refer to the treatments shown in $B-D$. The output of the disynaptic pathway could be blocked with strychnine, whereas the activation of this pathway could be blocked using high-calcium Ringer's solution to raise the spike threshold in the interposed neuron or using low-calcium Ringer's solution or glutamate receptor antagonists to reduce EIN-evoked EPSP amplitudes. Note that the latter treatments will affect the amplitude of direct EIN inputs to motor neurons (MN) and to the SilNs. The direct input will persist, but the delayed IPSP will be blocked because the reduced EPSP amplitude will not be able to evoke spiking in the SilN. B, Blocking the output of the proposed disynaptic pathway with strychnine (1 $\mu \mathrm{m})$ converted the depression of the direct EPSP into facilitation. C, High-calcium Ringer's solution blocked the delayed IPSP during the spike train, presumably by preventing the SilN from reaching spike threshold, and also abolished the depression of the direct EPSP. D, The non-NMDA glutamate receptor antagonist DNQX $(2 \mu \mathrm{m})$ reduced the amplitude of the direct EPSP in motor neurons and abolished the delayed IPSP, presumably by reducing the amplitude of the EIN-evoked EPSP in the SiIN. All traces are averages of at least five sweeps. Black traces show the control response, and gray traces show the effects of the different treatments. Graphs showing the amplitude $(E)$ and half-width $(F)$ of the direct EPSP in a motor neuron evoked by EIN stimulation at $20 \mathrm{~Hz}$ in control in the presence of the glycine receptor antagonist strychnine (1 $\mu \mathrm{m})$, high-calcium Ringer's solution, and the non-NMDA receptor antagonist DNQX (2 $\mu \mathrm{m})$.

calcium Ringer's solution could reflect a higher release probability in the disynaptic excitatory pathway (see Discussion). Blocking NMDA receptor-mediated inputs with AP-5 (100 $\mu \mathrm{M})$ (Alford and Grillner, 1990) reduced the amplitude of the direct and delayed excitatory input, but again neither component was 
A

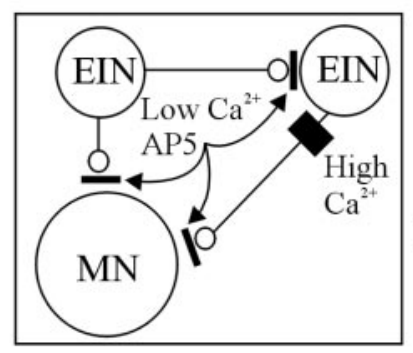

Bi
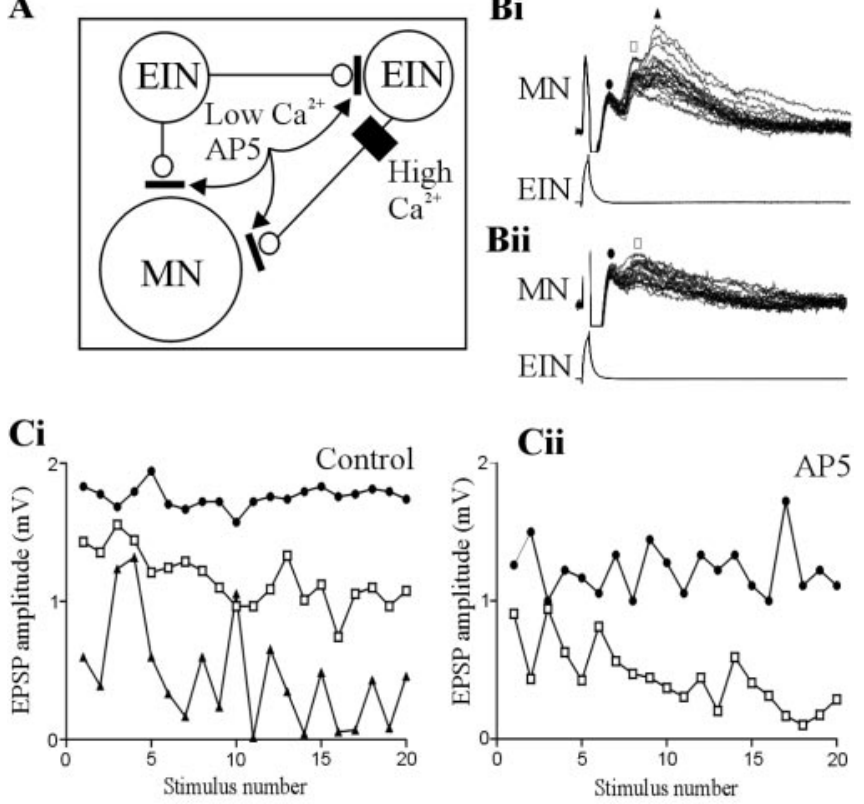

Di

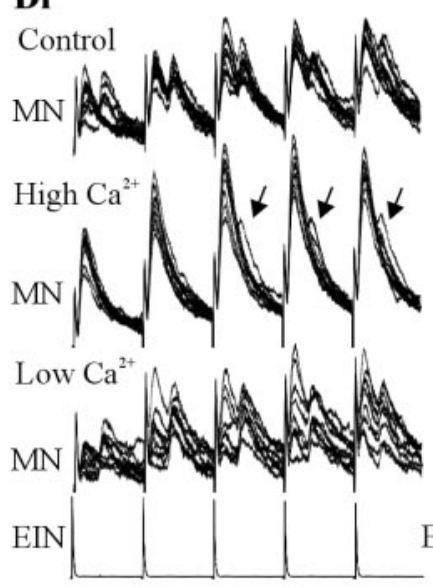

Dii

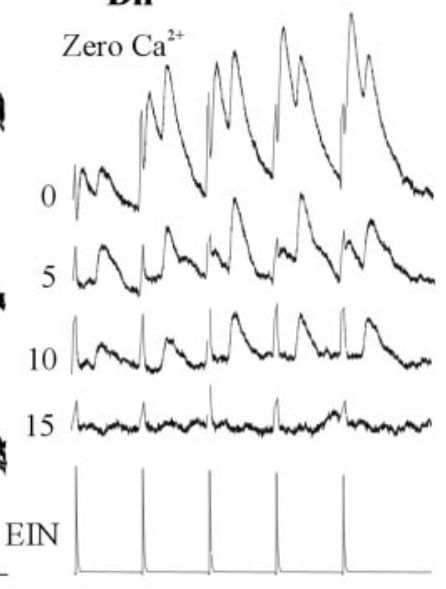

$1 \mathrm{mV}$
$60 \mathrm{mV}$

$\mathrm{Bi}, \mathrm{Bii} 20 \mathrm{~ms}$

Di,Dii $50 \mathrm{~ms}$

Figure 7. A, Summary of the proposed circuitry underlying the direct and delayed EPSPs and the methods used to examine the influence of the delayed EPSPs. Bi, An example of a triphasic feedforward EPSP in motor neurons evoked by stimulation of a single EIN. Bii, The triphasic, but not the biphasic, component was blocked by the NMDA receptor antagonist AP-5 (100 $\mu \mathrm{M})$. The symbols above the traces refer to the graphs in Ci and Cii, which show the change in the amplitude of the initial (circle), the biphasic (square), and the triphasic (triangle) excitatory input in response to $20 \mathrm{~Hz}$ stimulation of the presynaptic EIN in control (Ci) and in AP-5 (Cii). Note that the triphasic input was blocked. Di, The effect of changing Ringer's solution calcium levels on the delayed EPSP. High-calcium Ringer's solution increased the amplitude of the initial EPSP. It also reduced the reliability of the delayed EPSP but did not abolish it (arrows). Low-calcium Ringer's solution reduced the initial EPSP amplitude but again did not abolish the delayed input. Dii, Traces showing the effect of zero-calcium Ringer's solution over time. Note that the monosynaptic EPSP was abolished before the delayed EPSP. The numbers at the side of the traces shows the time in minutes after application of zero-calcium Ringer's solution. MN, Motor neuron.

abolished ( $n=4$ of 4 ) (Fig. $7 B, C$ ), although the triphasic excitatory input could be abolished by AP-5 ( $n=1$ of 2) (Fig. 7Bii).

Because the delayed EPSP was not blocked, its effect on the direct EPSP could not be determined. However, when the tripha- sic input shown in Figure 7Bi was blocked by AP-5, the activitydependent plasticity of the direct or biphasic EPSP was not altered (Fig. 7, compare $\mathrm{Ci}$, Cii), suggesting against an influence on the activity-dependent properties of the direct input.

\section{Discussion}

This study has shown that, in addition to their direct inputs in motor neurons, EINs in the lamprey locomotor network can evoke delayed feedforward IPSPs or EPSPs. The delayed IPSP depressed the direct EPSP over physiologically relevant spike trains and could thus contribute to the termination of ipsilateral motor neuron activity.

\section{Hemisegmental connectivity}

High-calcium Ringer's solution blocked the delayed IPSP, confirming that it was mediated polysynaptically, presumably through a divergent EIN input to an inhibitory interneuron (Berry and Pentreath, 1976). The SiINs, and not the LINs (Buchanan, 2001), were probably involved, because the delayed IPSP was associated with extracellular activity consistent with SiIN, but not LIN, activation, and it occurred in regions of the spinal cord in which the LINs are absent. A monosynaptic connection from the EINs to the SiINs was identified, which, together with the monosynaptic SiIN input to motor neurons (Buchanan and Grillner, 1988), provided the appropriate circuitry needed to evoke the delayed IPSP. Monosynaptic inhibitory connections from theSiINs to the EINs were also found. This could provide feedback inhibition of the EINs that would complement the feedforward inhibition of motor neurons in terminating hemisegmental network activity.

These connections add to the information available on the hemisegmental network (Buchanan, 2001) (Fig. 8A). However, the connectivity between hemisegmental networks is relatively poorly understood. For example, there is uncertainty over whether the large CC interneurons or the small crossing inhibitory interneurons (ScINs) mediate segmental reciprocal inhibition. Although the evidence suggests a role for the ScINs (Rovainen, 1983; Buchanan, 1999; Parker, 2000), there is no information on network interneuron inputs to the ScINs or on ScIN output connections to interneurons in the opposite hemisegment. In addition, although CC interneuron connectivity has been examined in detail (Buchanan, 1982), not all of the assumed CC interneuron connections (Grillner, 2003; Hill et al., 2003) have been verified experimentally (Parker, 2000; Buchanan, 2001). Finally, the relevance of reciprocal excitatory connections between hemisegmental networks is unknown.

\section{Properties in the disynaptic pathway}

Delayed feedforward IPSPs and EPSPs were evoked reliably over spike trains at frequencies of up to $20 \mathrm{~Hz}$. This is surprising because polysynaptic inputs usually fail at these frequencies (Berry and Pentreath, 1976). Specialized cellular or synaptic properties in the disynaptic pathways presumably allowed reliable feedforward inputs. The EIN-evoked EPSP was significantly larger in the SiINs than in motor neurons, and it usually facilitated in the SiINs but usually depressed in motor neurons (Parker, 2003). Facilitation from a relatively large initial EPSP will make the EIN input to the SiINs functionally stronger than the EIN input to motor neurons. This was supported by the ability of the EINs to evoke spikes in a relatively large proportion of the SiINs $(\sim 50 \%$ compared with 3\% in motor neurons) (Parker, 2003). There was no difference in the amplitude of the initial EPSP in SiINs that did or did not spike. There could, however, have been a difference in 
A

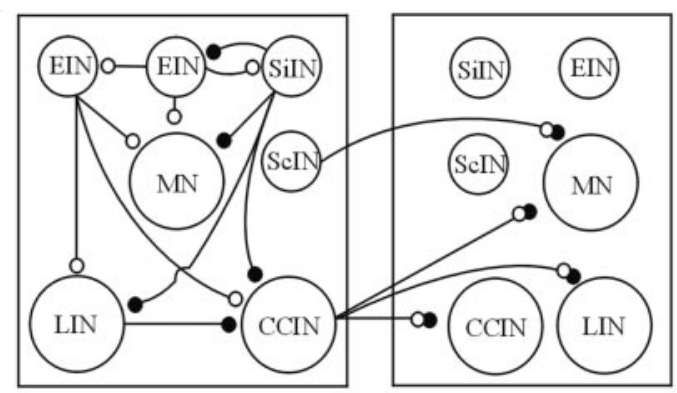

Bi

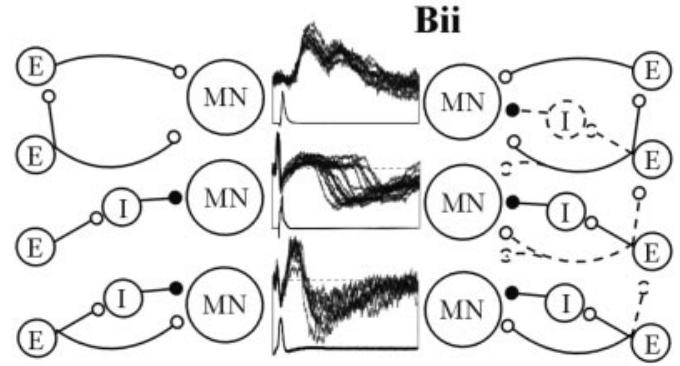

Figure 8. A, Summary of the connectivity in the locomotor network, to include the connections identified here. Open circles indicate excitatory glutamatergic connections, and filled circles indicate inhibitory glycinergic connections. Mixed symbols shows interneuron inputs that can be either inhibitory or excitatory. Only connections that have been verified experimentally are shown. Bi, Diagram showing how different functional assemblies could result from anatomically fixed connections made by individual cells in the EIN pool. Bii, Diagram showing how assemblies could form from a pool of ElNs with common synaptic connections. The solid lines show the cells and connections that would be selected to evoke the functional assemblies shown here. The traces in the center show examples of the different feedforward effects identified here. E, Excitatory inputs; I, inhibitory inputs; CCIN, crossed caudal interneuron; MN, motor neuron.

the degree of facilitation, but this was not examined. EIN-evoked spiking in motor neurons seems to reflect a cellular property of the postsynaptic motor neuron (Parker, 2003), and cellular properties could also contribute to the EIN-evoked spiking in the SiINs.

The SiIN input to motor neurons usually depresses (Fig. 5G). However, SiINs that received facilitating EIN inputs made facilitating connections onto motor neurons. Facilitation thus usually occurs at both synapses in the disynaptic pathway. Facilitation will gradually increase the strength of the IPSP and could contribute to the delayed depression of the direct EPSP during spike trains.

Although the sample size of EIN to SiIN connections is relatively small, the correlation of EIN and SiIN activity-dependent properties (Fig. $5 F$ ) suggests that the presynaptic EIN could influence SiIN output synapses. A transynaptic effect (Fitzsimonds et al., 1997) could account for the variability of EIN inputs to motor neurons (Parker, 2003), which were examined without information on the inputs to the EINs. Although it would help to explain the variability in synaptic properties, transynaptic effects would complicate the analysis of network synapses, because paired recordings from monosynaptically connected cells alone would not be sufficient to characterize connections: information would also be required on the wider interactions of the neurons studied.

The influence of the delayed IPSP was most pronounced during the spike train. However, in some cases, effects occurred on the initial EPSP (Fig. 6B). This could reflect low-frequencyevoked delayed IPSPs on initial EPSPs, which occurred in a small proportion of connections. In addition, strychnine can broaden action potential through by affecting potassium channels (Rayport and Kandel, 1981), and this could have contributed to its effects. Together, however, the analysis of the disynaptic pathway suggests an effect of the delayed IPSP on direct inputs to motor neurons.

\section{Functional network assemblies}

The delayed IPSP occurred in $\sim 10 \%$ of EIN to motor neuron connections and the delayed EPSP in $\sim 3 \%$ of connections. Divergent connections could be more widespread, but they may either not be sufficient to activate interposed neurons on their own or the feedforward output may not be made onto the postsynaptic neuron examined.

There are $\sim 50$ EINs in the hemisegmental network (Buchanan and McPherson, 1995). The delayed excitatory and inhibitory effects shown here may reflect unique anatomically fixed assemblies made by a proportion of the cells in the EIN pool (Fig. $8 \mathrm{Bi}$ ). These could be selected by sensory or descending inputs (Zelenin et al., 2001) to alter the balance of excitation and inhibition during network activity. Alternatively, all of the cells in the EIN population could have the same connectivity, different functional assemblies forming through neuromodulator or activity-dependent selection of particular connections (Fig. 8 Bii) (Parker, 2003).

Because individual EINs cannot be uniquely identified, it is difficult to know whether different EINs have different patterns of synaptic connections. The ability to block the disynaptic pathway shows that cellular or synaptic properties can influence functional assemblies. However, novel assemblies are not revealed when cellular or synaptic properties are altered by changing Ringer's solution calcium or potassium levels, by blocking inhibitory or excitatory synaptic transmission, or by neuromodulators. This suggests the presence of anatomically fixed assemblies made by a proportion of cells in the EIN pool and argues against the stereotyped connectivity often assumed in network models.

The delayed EPSP was less sensitive to glutamate receptor antagonists or changes in calcium levels than the delayed IPSP. The persistence of the delayed EPSP in AP- 5 ruled out the possibility that it reflected a late NMDA component to the direct EPSP. The initial EPSP was not an electrical component, because it was reduced by AP-5. It is unlikely that the delayed EPSP was caused by the asynchronous release of vesicles from a single EIN ( $\mathrm{Lu}$ and Trussell, 2000), because delayed EPSP amplitudes were considerably larger than the quantal EPSP amplitude (Parker, 2003). The delayed EPSP instead presumably reflected feedforward connections between EINs (Parker, 2000). Although its persistence in high- or low-calcium Ringer's solution or AP-5 suggests functionally strong connections between the EINs, this is not supported by the available data, which suggests that this connection depresses from a relatively small $(\sim 1 \mathrm{mV})$ initial EPSP (Parker, 2000) (Fig. 4C). The analysis of other network synapses, however, shows that specific connections do not have single defining characteristics but a distribution of activity-dependent properties (Parker, 2000, 2003). Additional analyses of connections between EINs could thus reveal properties that are consistent with the feedforward excitatory effects suggested here. EINs can make electrical synapses onto motor neurons (Parker, 2003). An electrical connection between EINs could account for the persistence of the delayed EPSP when glutamatergic transmission was reduced and the partial inhibitory effect of high-calcium Ringer's solution (Giaume and Venance, 1998), effects that should abolish chemical transmission in polysynaptic pathways. 


\section{Function of the delayed IPSP}

If EIN inputs were examined without identifying the SiIN or if the wider network interactions of the SiIN were unknown, facilitation at this connection would be interpreted as an increase in ipsilateral excitatory drive, not the reduction that results from the feedforward inhibition. This emphasizes that cellular and synaptic effects can only be understood by placing identified cells in the context of identified network interactions. Functional effects will ideally be examined during network activity. Unfortunately, the large number of synaptic inputs that postsynaptic cells receive during network activity has so far complicated this analysis (my unpublished observations).

The feedforward IPSP depressed direct EPSPs during physiologically relevant spike trains. The IPSP developed after $\sim 12$ msec. It will thus coincide with the NMDA component of direct EPSPs (Dale and Grillner, 1986). A reduced NMDA component will reduce the amplitude of individual EPSPs, and, by reducing the EPSP duration, it will limit EPSP summation.

The feedforward inhibition will be influenced by the strength of transmission in the disynaptic pathway. This will be influenced by the degree of facilitation at both synapses in the disynaptic pathway, which will introduce a delayed increase in the strength of the feedforward inhibition. Homosynaptic activity-dependent plasticity acting on heterosynaptic network interactions could thus influence the integration of synaptic inputs during network activity.

\section{References}

Alford S, Grillner S (1990) CNQX and DNQX block non-NMDA synaptic transmission but not NMDA-evoked locomotion in lamprey spinal cord. Brain Res 506:297-302.

Berry M, Pentreath V (1976) Criteria for distinguishing between monosynaptic and polysynaptic transmission. Brain Res 105:1-20.

Buchanan J (1993) Electrophysiological properties of identified classes of lamprey spinal neurons. J Neurophysiol 70:2313-2325.

Buchanan J (1999) Commissural interneurons in rhythm generation and intersegmental coupling in the lamprey spinal cord. J Neurophysiol 81:2037-2045.

Buchanan J (2001) Contributions of identifiable neurons and neuron classes to lamprey vertebrate neurobiology. Prog Neurobiol 63:441-466.

Buchanan J, Cohen AH (1982) Activities of identified interneurons, motoneurons, and muscle fibers during fictive swimming in the lamprey and effects of reticulospinal and dorsal cell stimulation. J Neurophysiol 47:948-960.

Buchanan J, Grillner S (1988) A new class of small inhibitory interneurones in the lamprey spinal cord. Brain Res 438:404-407.

Buchanan J, Kasicki S (1995) Activities of spinal neurons during brain stemdependent fictive swimming in lamprey. J Neurophysiol 73:80-87.

Buchanan J, McPherson D (1995) The neuronal network for locomotion in the lamprey spinal cord: evidence for the involvement of commissural interneurons. J Physiol (Paris) 89:221-233.

Buchanan J, Grillner S, Cullheim S, Risling M (1989) Identification of excitatory interneurons contributing to generation of locomotion in lamprey: structure, pharmacology, and function. J Neurophysiol 62:59-69.

Buchanan JT (1982) Identification of interneurons with contralateral, caudal axons in the lamprey spinal cord: synaptic interactions and morphology. J Neurophysiol 47:961-975.

Buchanan JT, Kasicki S, Quinlan KA (1998) Effects of motoneuron stimulation in the lamprey spinal cord. Soc Neurosci Abstr 24:1666.
Buszaki G (1984) Feedforward inhibition in the hippocampal formation. Prog Neurobiol 22:131-153.

Cochilla A, Alford S (1999) NMDA receptor-mediated control of presynaptic calcium and neurotransmitter release. J Neurosci 19:193-205.

Dale N, Grillner S (1986) Dual-component synaptic potentials in the lamprey mediated by excitatory amino acid receptors. J Neurosci 6:2653-2661.

Eccles JC, Fatt P, Koketsu K (1954) Cholinergic and inhibitory synapses in a pathway from motor axon collaterals to motoneurones. J Physiol (Lond) 126:524-562.

Fitzsimonds R, Song H-J, Poo M-M (1997) Propagation of activitydependent synaptic depression in simple neural networks. Nature 388:439-448.

Getting P (1989) Emerging principles governing the operation of neural networks. Annu Rev Neurosci 12:185-204.

Giaume C, Venance L (1998) Intercellular calcium signaling and gap junctional communication in astrocytes. Glia 24:50-64.

Grillner S (2003) The motor infrastructure: from ion channel to neuronal networks. Nat Rev Neurosci 4:573-586.

Hill AVH, Masino MA, Calabrese RL (2003) Intersegmental coordination of rhythmic motor patterns. J Neurophysiol 90:531-538.

Jonas P, Bischofberger J, Sandkuhler J (1998) Corelease of two fast neurotransmitters at a central synapse. Science 281:419-424.

Lu T, Trussell L (2000) Inhibitory transmission mediated by asynchronous transmitter release. Neuron 26:683-694.

Marder E, Calabrese R (1996) Principles of rhythmic motor pattern generation. Physiol Rev 76:687-717.

McPherson D, Buchanan J, Kasicki S (1994) Effects of strychnine on ficitive swimming in the lamprey: evidence for glycinergic inhibition, discrepancies with model predictions, and novel modulatory rhythms. J Comp Physiol [A] 175:311-321.

Miles R (1990) Synaptic excitation of inhibitory cells by single CA3 hippocampal pyramidal cells of the guinea-pig in vitro. J Physiol (Lond) 428:61-77.

Nadim F, Manor Y (2000) The role of short-term synaptic dynamics in motor control. Curr Opin Neurobiol 10:683-690.

Parker D (2000) Spinal cord plasticity: independent and interactive effects of neuromodulator and activity-dependent plasticity. Mol Neurobiol 21:1-26.

Parker D (2003) Variable properties in a single class of excitatory spinal synapse. J Neurosci 23:3154-3163.

Parker D, Grillner S (2000) The activity-dependent plasticity of segmental and intersegmental synaptic connections in the lamprey spinal cord. Eur J Neurosci 12:2135-2146.

Rayport S, Kandel E (1981) Epileptogenic agents enhance transmission at an identified weak electrical synapse in Aplysia. Science 213:462-463.

Rovainen C (1974) Synaptic interactions of identified nerve cells in the spinal cord of the sea lamprey. J Comp Neurol 154:189-206.

Rovainen C (1982) Neurophysiology. In: The biology of lampreys (Hardisty M, Potter I, eds), pp 1-136. London: Academic.

Rovainen C (1983) Identified neurons in the lamprey spinal cord and their roles in fictive swimming. In: Neural origin of rhythmic movements. (Roberts A, Roberts BL, eds), pp 305-330. Cambridge, UK: Cambridge UP.

Safronov BV, Baev KV, Batueva IV, Rusin KI, Suderevskaya EI (1989) Peculiarities of receptor-channel complexes for inhibitory mediators in the membranes of lamprey spinal cord neurones. Neurosci Lett 102:82-86.

Selverston A (1980) Are central pattern generators understandable. Behav Brain Sci 3:535-571.

Wallén P, Lansner A (1984) Do the motoneurones constitute apart of the spinal network generating the swimming rhythm in the lamprey. J Exp Biol 113:493-497.

Zelenin PV, Grillner S, Orlovsky GN, Deliagina TG (2001) Heterogeneity of the population of command neurons in the lamprey. J Neurosci 21:77937803. 